\title{
Urostomy Leak, CTCAE
}

National Cancer Institute

\section{Source}

National Cancer Institute. Urostomy Leak, CT CAE. NCI Thesaurus. Code C143910.

A finding of leakage of contents from a urostomy. 